\title{
Frontières, asile et détention : la fabrique locale de la frontière
}

\author{
Mathilde Darley
}

\section{Lieux-frontières}

Un poste-frontière. Sur la frontière Schengen qui sépare encore, au printemps 2007, l'Autriche de la République tchèque, un centre de coopération policière entre les polices tchèque et autrichienne est en cours d'installation. En tant qu'« étudiante française en sociologie travaillant sur le contrôle aux frontières ", je suis autorisée par le ministère de l'Intérieur autrichien à y séjourner une semaine à deux reprises. Le poste est contrôlé nuit et jour, de part et d'autre de la frontière, par des policiers des deux pays et un important dispositif de surveillance (reposant notamment sur l'utilisation de caméras à thermovision) est déployé pour contrôler la « frontière verte », portion de frontière entre deux postes de contrôle. Les deux polices mettent par ailleurs en place des patrouilles communes, qui ont pour objectif de sécuriser la frontière verte. Les populations frontalières sont incitées par des campagnes de presse à participer au contrôle en signalant à la police toute présence jugée suspecte. En marge de cette représentation policière et médiatique de la frontière comme un espace de contrôle technicisé, Johnny, ancien policier, m'explique comment son observation fine des cycles de travail policiers et sa connaissance des logiques de suspicion aux frontières lui a permis de faire passer 150 migrants $^{1}$ en une vingtaine de voyages, à bord de son véhicule, depuis la République tchèque vers l'Italie.

Un centre pour demandeurs d'asile. Situé dans la zone de transit d'un aéroport international, en sous-sol, gardé par des policiers, il est destiné au confinement des personnes qui déclarent vouloir demander l'asile à leur descente d'avion en République tchèque. Les demandes d'asile déposées ici font l'objet d'un examen accéléré destiné à écarter rapidement les demandes de protection jugées manifestement infondées ${ }^{2}$ et d'en renvoyer les auteurs vers leur pays d'origine ou de transit. L'ONG à laquelle l'Etat a délégué l'assistance aux migrants assure une permanence juridique hebdomadaire pour les migrants en exprimant le souhait. En pratique, les demandeurs sont pour la plupart dirigés vers un centre ouvert pour demandeurs d'asile situé sur le territoire national à l'expiration de la durée maximale d'enfermement (42 jours au moment de l'enquête), car l'autorité en charge de l'examen des demandes d'asile ne parvient pas à statuer sur leur demande dans le délai imparti. Dans le couloir du centre fermé, un téléphone commun sonne

\footnotetext{
${ }^{1}$ L'écriture inclusive n'a finalement pas été conservée dans ce texte pour des raisons de longueur du manuscrit. A chaque occurrence, il convient donc de lire migrant.e.s, policier.e.s, demandeurs.euses, aumônier.e.s, etc.

2 Face à l'augmentation des demandes d'asile sur le territoire européen au début des années 1990, à la suite notamment des conflits en ex-Yougoslavie et du démantèlement du «bloc de l'Est », les Etats membres de l'Union européenne se dotent d'instruments permettant de légaliser l'examen et le rejet accélérés de certaines demandes. La résolution adoptée le 30 novembre 1992 par les ministres de l'immigration des pays membres définit ainsi comme «infondées» les demandes de protection à propos desquelles l'officier de protection estime que la crainte du demandeur d'être persécuté dans son propre pays est «dénuée de fondement» ou que le demandeur peut «trouver une protection efficace dans une autre partie de son pays ». La crainte est notamment considérée comme «infondée » si le requérant est ressortissant d'un Etat figurant sur la liste des pays dits «sûrs » établie par chacun des Etats membres de l'UE.
} 
régulièrement, provoquant l'attroupement : c'est sur ce numéro que les migrants transférés vers un centre ouvert et ayant réussi à poursuivre leur voyage vers les pays situés plus à l'Ouest de l'Europe informent ceux qui sont encore enfermés sur les routes migratoires empruntées et les passeurs à contacter. Chargée par l'ONG intervenant dans le centre d'y organiser des ateliers photos, je rencontre Luther, originaire du Cameroun, qui a détruit ses documents de voyage (établis au nom d'un autre) avant de se présenter aux autorités tchèques pour demander l'asile. L'examen de sa demande d'asile, motivée par les persécutions politiques dont Luther dit avoir été victime au Cameroun, est identifiée par l'administration comme requérant un soin particulier et Luther est donc rapidement transféré du centre de réception de l'aéroport vers un centre ouvert pour demandeurs d'asile. Au cours des contacts que nous maintenons après son transfert en centre ouvert, Luther déclare s'appeler en réalité William. Au printemps, il m’informe qu'il est passé clandestinement en Italie et se trouve désormais à Naples. Lorsque je lui rends visite à Naples à l'été 2006, ses colocataires l'appellent Paul. Un an plus tard, c'est à Paris qu'il me donne rendez-vous, où il travaille et vit sous un nouveau nom, grâce au prêt de papiers d'identité par un compatriote.

Un centre de rétention. Isolé dans la campagne tchèque, au milieu des bois, à cinq kilomètres du village (lui-même desservi par un bus permettant deux fois par jour d'atteindre la prochaine gare), le centre est entouré de hauts barbelés et gardé par des policiers. A l'intérieur, des agents de sécurité privés surveillent les étages où sont retenus les étrangers interpellés en situation irrégulière et à l'encontre desquels une procédure d'expulsion du territoire est en cours. Les travailleurs sociaux, employés du ministère des Affaires sociales, y ont également des bureaux dans lesquels ils reçoivent régulièrement les étrangers pour suivre l'évolution de leur dossier. En tant qu' étudiante en travail social bénéficiant d'une autorisation de stage du ministère des Affaires sociales », je suis hébergée dans le centre pendant une semaine et j'accompagne les travailleurs sociaux. Sont enfermés ici des hommes et des femmes seul.e.s, mais aussi des familles avec enfants. Pour ces derniers, une garderie est située dans un des bâtiments sa façade colorée et les dessins d'enfants accrochés aux fenêtres. Une fois par semaine, un représentant d'ONG vient proposer une assistance juridique aux étrangers qui souhaitent faire valoir leur droit à une forme de régularisation du séjour. Madame S., originaire de Somalie, est retenue ici depuis plusieurs mois. Son nourrisson, âgé d'un mois, est né en rétention. Dans sa chambre plongée dans l'obscurité, elle gémit de longues heures durant sur son lit. Les travailleurs sociaux expliquent que son expulsion est compliquée par le fait qu'elle ne comprend qu'un dialecte somalien pour lequel les interprètes sont rares et coûteux, ce qui ralentit son identification par les autorités, et qu'elle ne « coopère pas » en refusant de parler. Ils reprochent de manière générale à Madame $S$. de ne pas accepter « l'aide qui lui est proposée », et notamment de refuser l'alimentation spéciale qui lui est distribuée pour son nourrisson. Dans sa chambre, ils déclarent à mon intention : "Les gens comme ça, il faut les expulser, on veut les aider et ils ne trouvent rien d'autre à faire que de jeter ce qu'on leur donne! $\rangle^{3}$.

Poste-frontière, centre fermé pour demandeurs d'asile, centre de rétention : trois lieux où

\footnotetext{
3 Les extraits entre guillemets émaillant le texte sont tirés d'entretiens conduits avec des acteurs non gouvernementaux, des travailleurs sociaux, des policiers, des officiers de protection de l'asile, des migrants et des passeurs en Autriche, Allemagne et République tchèque entre 2005 et 2013.
} 
s'exerce, donc, cette " autorité souveraine de l'Etat d'exclure ${ }^{4}$ certains étrangers de son territoire national. Trois «lieux-frontières", pourrait-on dire, puisque les politiques contemporaines d'immigration et de sécurité leur confèrent de facto une fonction centrale de filtrage et de « tri » des populations étrangères autorisées ou non à pénétrer ou séjourner sur le territoire national. Trois lieux, surtout, où se donnent à voir le contrôle des migrations à travers les dispositifs protéiformes au sein desquels il s'incarne, les multiples acteurs qu'il fait intervenir, les logiques contrastées dans lesquelles il s'inscrit et les formes de pouvoir/contre-pouvoir qui y prennent corps. Entre «gardiens » et "passeurs » de frontières, entre dispositifs de contrainte et prise en charge humanitaire, entre imposition d'un pouvoir étatique de contrôle et contournements locaux, cette contribution s'attachera donc à penser ensemble des lieux et des acteurs de la frontière que l'analyse sociologique a souvent cloisonnés. Prisonnière d'une forme de « nationalisme méthodologique » plaçant l'Etat-nation au centre de l'attention, la sociologie a en effet longtemps achoppé sur le constat d'un décalage entre les intentions premières des politiques étatiques de régulation des migrations et leurs effets concrets ${ }^{5}$, ainsi que sur une querelle scientifique entre les auteurs arguant d'un pouvoir étatique croissant en matière de contrôle des mobilités ${ }^{6}$, et ceux s'attachant au contraire à présenter comme déclinante la capacité des Etats à contrôler les frontières de leur territoire ${ }^{7}$. Décaler le regard sociologique pour s'intéresser aux lieux de contrôle " par le bas » me semblait donc permettre de dépasser l'impasse à laquelle avait pu conduire la focalisation sur l'Etat-nation comme seule instance de contrôle. Pour ce faire, je me suis appuyée sur des observations et entretiens réalisés au sein de ces différents lieuxfrontières. Ces enquêtes m'ont conduite à endosser différents rôles, de celui de bénévole pour différentes associations en charge des loisirs et/ou de l'accompagnement juridique dans les lieux d'enfermement, à celui d'étudiante réalisant un stage d'observation du «travail social en milieu fermé », en passant par interprète, organisatrice d'ateliers de photographie ou aumônière stagiaire. Ces différentes «casquettes» répondaient non seulement à la nécessité de s'adapter aux contraintes présidant à l'entrée sur les lieux d'enquête, mais aussi à celle que je m'étais fixée de faire varier les points de vue pour mettre en lumière la «fabrique locale de la frontière », des formes concrètes de sa mise en œuvre à celles de son éventuel contournement.

Si les développements de la première partie visent à remettre en question la frontière et son contrôle comme une construction purement juridique, parfaitement « traçable » et linéaire dans sa matérialisation, les enquêtes conduites en différents lieux de contrôle invitent également à complexifier l'analyse des effets du contrôle sur celles et ceux qui cherchent à franchir les frontières, en dépassant l'appréhension des lieux-frontières comme des lieux de pouvoir opposant le groupe restreint des "gardiens » à la masse des personnes soumises au pouvoir étatique de contrôle. Dans quelle mesure ces lieux-frontières offrent-ils la possibilité de marges de

\footnotetext{
${ }^{4}$ Geddes Andrew, « Europe's Border Relationships and International Migration Relations », Journal of Common Market Studies, 43, 4, 2005, p.787-806.

5 Voir Cornelius Wayne A., Martin Philip L., Hollifield James F., «Introduction : The Ambivalent Quest for Immigration Control », in Cornelius Wayne A., Martin Philip L., Hollifield James F. (dir.), Controlling Immigration : A Global Perspective, Stanford (Californie, US) : Stanford University Press, 1994.

"Par exemple Freeman Gary, "Can Liberal States Control Unwanted Migration ?", The Annals of the American Academy of Political and Social Science, vol. 354, 1994, p. 17-30 ; Lahav Gallya, "The Rise of Non-State Actors in Migration Regulation in the United States and Europe : Changing the Gatekeepers or "Bringing Back the State" », in Foner Nancy, Rumbaut Ruben, Gold Steven (dir.), Transformations: Immigration and Immigration Research in the United States, New York : Russell Sage Foundation, 2000.

${ }^{7}$ Sassen Saskia, Losing Control? Sovereignty in an Age of Globalization, New York : Columbia University Press, 1996.
} 
manœuvre aux migrants leur permettant de contourner certaines des règles du jeu dictées par les Etats ? Si elles existent, ces pratiques échappant aux "gardiens » permettent-elles de limiter le pouvoir d'imposition de la frontière, voire d'en transformer le tracé ?

\section{Tracer la frontière, une prérogative régalienne?}

Dès le $18^{\mathrm{e}}$ siècle, la nationalisation des frontières en Europe va de pair avec la mise en place d'une distinction entre citoyens et étrangers, la possibilité d'expulser les étrangers constituant à ce titre une technique cruciale de "clôture du territoire ». A partir des années 1970 cependant, dans un contexte marqué par la crise économique et le durcissement des politiques d'immigration des Etats européens, le tracé de la frontière entre citoyens et étrangers, et plus encore entre étrangers dont la présence sur le territoire national est autorisée ou tolérée, et étrangers devant faire l'objet d'une mesure d'expulsion, fait l'objet d'une repolitisation. Au tournant des années 1990, l'effondrement de l'ancien bloc socialiste, le conflit en ex-Yougoslavie, et les mouvements accrus de populations auxquels ils donnent lieu, mais aussi le déclin du modèle de l'asile tel qu'il avait émergé au sortir de la Seconde Guerre mondiale, marquent l'avènement d'une politique migratoire européenne fondée sur un contrôle renforcé des frontières ainsi que sur la systématisation du recours à l'enfermement des étrangers ne satisfaisant pas aux critères d'entrée ou de séjour sur le territoire national et devant donc en être expulsés. Si les Accords de Schengen signés en $1985^{\circ}$ prévoient certes la libre circulation des ressortissants communautaires, la Convention d'application de ces mêmes Accords, signée en 1990 et entrée en vigueur en 1995, procède de l'idée que l'Europe de la libre circulation ne saurait se réaliser sans que soit adoptée une série de mesures dites "compensatoires », venant contrebalancer le déficit de sécurité et le risque d' "invasion migratoire» censés résulter de l'ouverture des frontières internes à l'espace Schengen ${ }^{10}$. Parmi ces mesures, figurent notamment un durcissement des conditions d'accès à la demande d'asile, avec l'adoption du principe de détermination d'un Etat unique responsable de l'examen de la demande de protection, ainsi qu'un renforcement des contrôles aux frontières extérieures de l'espace Schengen ${ }^{11}$. Autrement dit, tandis que s'estompent certaines frontières sur le territoire européen, d'autres au contraire se font plus saillantes, témoignant s'il en était besoin de la dimension symbolique et matérielle que conserve le contrôle aux frontières comme rempart de la souveraineté ${ }^{12}$. Les travaux de sciences sociales s’intéressant aux formes du contrôle étatique

\footnotetext{
${ }^{8}$ Brubaker Rogers, Citoyenneté et nationalité en France et en Allemagne, Paris, Belin, 1997.

${ }^{9}$ L'accord de Schengen est signé le 14 juin 1985 par la France, la République Fédérale d’Allemagne et le Benelux. Il entre en vigueur en 1995, avec la Convention d'application de l'accord de Schengen, et intégré à l'acquis communautaire avec le Traité d'Amsterdam de 1997. Il s'applique désormais à tous les Etats membres de l'espace Schengen (soit aujourd'hui 26 Etats, dont 22 membres de l'Union européenne), mais aussi à tous les Etats candidats à l'entrée dans l'UE.

${ }^{10}$ Bigo Didier (dir.), L'Europe des polices et de la sécurité intérieure, Bruxelles, Editions Complexe, 1992.

${ }^{11}$ Les instruments de ce renforcement des contrôles incluent notamment la mise en place de fichiers informatiques uniformisés (Schengen Information System), le développement de la coopération policière et judiciaire visant à lutter contre la criminalité transfrontalière, mais aussi la création d'une zone dite "zone Schengen » autorisant les contrôles d'identité sans justification dans une bande de 20 kilomètres de part et d'autre des frontières Schengen, ou encore, une dizaine d'année plus tard, la création de l'agence Frontex (2004), visant à coordonner la gestion des frontières externes des Etats membres de l'UE.

${ }^{12}$ Comme est venu le rappeler également le rétablissement des contrôles aux frontières au sein de l'espace Schengen après ladite « crise des réfugiés » en 2015.
} 
des mouvements de populations se développent alors, et les frontières y sont souvent présentées comme un espace privilégié d'investigation et d'interprétation des aspects symboliques de l'Etat ${ }^{13}$.

M'intéressant ici aux pratiques locales de tracé des frontières, je me concentrerai en particulier sur deux points nodaux de cette compétence régalienne à trier les circulations et à distinguer les étrangers autorisés à entrer et/ou rester sur le territoire des autres : le contrôle aux frontières, à partir d'une enquête conduite dans un poste-frontière situé sur la frontière tchéco-autrichienne à l'époque (2006-2007) où celle-ci constituait encore une frontière externe de l'espace Schengen ${ }^{14}$; et l'asile, en m'appuyant sur des observations réalisées dans deux centres fermés aéroportuaires visant à retenir les demandeurs d'asile arrivés par avion, afin de mettre en œuvre un examen accéléré de leurs demandes de protection. Au sein de chacun de ces lieux œuvrent des professionnels de la frontière, mandatés par l'Etat pour donner corps aux limites nationales en désignant ceux qui sont autorisés à les franchir et ceux qui doivent au contraire rester « de l'autre côté ». C'est donc des pratiques de «tri » qu'ils mettent en œuvre dans leur travail quotidien de " gardiens des frontières » que je tenterai, dans cette première partie, de rendre compte.

\section{"Bien sûr, c'est un hasard si un migrant illégal passe justement par là au moment où nous contrôlons"}

Dès le début des années 1990, dans un contexte médiatique agitant la menace d'une «invasion » de l'Autriche par les flux migratoires en provenance d'ex-Yougoslavie et des anciens Etats socialistes, ce petit pays, alors candidat à l'entrée dans l'Union européenne, situé au carrefour entre deux Europe, celle «de l'Ouest» et celle «de l'Est»), mais se considérant déjà comme le dernier rempart de « l'Ouest », fait du contrôle aux frontières un élément central, voir « bruyant », de sa politique migratoire. Alors que les Etats post-communistes voisins s'attachent à démilitariser le contrôle des frontières, le Conseil des ministres autrichien décide, sur proposition du ministère de l'Intérieur, de stationner des soldats de l'armée fédérale le long de la frontière entre l'Autriche et la Hongrie pour « contrer l'augmentation du nombre de réfugiés économiques et réduire sensiblement les problèmes de police liés aux étrangers, à l'asile et à la criminalité constatés dans le contexte des franchissements illégaux des frontières ». Cette décision, à l'origine exceptionnelle, est depuis lors prolongée chaque année, pérennisant ainsi la participation de l'armée au contrôle de 455 kilomètres de frontières avec la Slovaquie et la Hongrie. Au-delà de la dimension symbolique forte que revêt alors la présence de militaires aux frontières autrichiennes, alors que s'ouvrent les frontières des Etats voisins sortis du communisme, le renforcement des contrôles fait également l'objet d'une médiatisation importante, établissant une corrélation directe entre immigration et criminalité. La presse relaie par ailleurs largement les appels lancés par l'armée et la police à l'adresse des populations des régions frontalières, incitées par le biais de tracts à participer à la protection des frontières et à la lutte contre l'immigration. Cette implication des habitants dans le contrôle des frontières s'inscrit ensuite dans la durée, les informations transmises par les populations frontalières restant présentées comme indispensables au travail des gardes-frontières autrichiens rencontrés à la fin des années 2000.

De l'autre côté de la frontière, après quarante ans de régime communiste marqué par différentes

\footnotetext{
13 Voir notamment le développement du courant de recherche des « border studies », et par exemple Wilson Thomas M., Donnan Hastings (eds.), Border Identities. Nation and State at International Frontiers, Cambridge, Cambridge University Press, 1998.

${ }^{14}$ La République tchèque a rejoint l'espace Schengen le 21 décembre 2007, tout comme la Slovaquie, la Slovénie, la Pologne, la Hongrie, la Lituanie, la Lettonie, l'Estonie et Malte.
} 
formes d'héroïsation du garde-frontière, la rupture politique de 1989 en Tchécoslovaquie conduit au contraire à faire du contrôle frontalier un élément relativement silencieux de la politique d'immigration qui se met en place au début des années 1990. Après son indépendance en 1993, et la déclaration de sa candidature à l'entrée dans l'UE en 1996, la République tchèque doit toutefois faire face à des pressions croissantes des pays voisins (Allemagne et Autriche), puis de l'Union européenne, érigeant le contrôle frontalier en espace de démonstration de sa capacité à assurer la sécurité de son territoire et, plus largement, des frontières de l'UE. En outre, à partir de 2004 et l'entrée de la République tchèque dans l'UE, cette portion de frontière tchéco-autrichienne est soumise à une "pression migratoire » accrue liée à l'afflux de migrants ayant fui la Tchétchénie, arrivant par la Pologne en République tchèque et cherchant à gagner l'Autriche pour y déposer une demande d'asile. C'est dans ce contexte qu'est décidée la mise en place d'un centre de coopération policière tchéco-autrichien à la frontière, dont l'ouverture en 2006 fait elle aussi l'objet d'une médiatisation importante mettant l'accent sur l'efficacité accrue permise par la mise en place de contrôles conjoints des deux polices, ainsi que sur la sophistication des équipements disponibles pour détecter les franchissements irréguliers à la frontière : caméras à thermo-vision installées le long de la frontière «verte », hélicoptères permettant le survol des zones frontalières, etc.

Les observations réalisées auprès des policiers aux frontières tchèques et autrichiens mettent pourtant à l'épreuve cette représentation géopolitique et médiatique de la frontière comme un espace de contrôle réduisant à son minimum le poids du hasard, un « rempart » technicisé contre les entrées indésirables sur le territoire autrichien, et plus largement au sein de l'espace Schengen. Au-delà des variations horaires (en fonction du nombre de policiers en poste, mais aussi de la suspicion plus importante dont font l'objet les franchissements nocturnes) et saisonnières (la végétation printanière et estivale compliquant le contrôle de la frontière "verte »), les observations révèlent néanmoins certaines constantes dans les formes différenciées de contrôle auxquelles les voyageurs sont soumis ${ }^{15}$. Les hommes sont ainsi plus systématiquement contrôlés que les femmes, les personnes seules plus que les couples et les voyageurs plus jeunes davantage que leurs homologues plus âgés. Les voitures immatriculées dans l'un des deux pays frontaliers et/ou dont le contrôle parle tchèque ou allemand font également l'objet d'un contrôle plus relâché, tandis que la présentation de documents d'identité «étrangers » et, surtout, la présentation de signes extérieurs d' "altérité», tels que la couleur de peau notamment, augmentent significativement les suspicions policières. Il en va de même pour les contrôles opérés par des unités communes patrouillant le long de la frontière verte, dont la mise en place a reçu un écho médiatique fort mais dont l'essentiel de l'activité repose sur des échanges linguistiques entre policiers tchèques et autrichiens, en quête de langue commune, et sur le contrôle sporadique d'individus jugés suspects principalement en raison de leur couleur de peau, rendant incongrue aux yeux des policiers leur présence dans les zones rurales frontalières. Interrogés sur ces variations, les policiers invoquent leur «flair», leur « instinct», ou encore leur «sixième sens» permettant de détecter les voyageurs «suspects». Leurs pratiques attestent pourtant avant tout de la prégnance d'une pensée par «groupes» et, plus encore, par «stéréotypes» présidant au contrôle des frontières et conduisant les gardiens des frontières (corps composé exclusivement d'hommes au moment de l'enquête), à associer un individu à une

\footnotetext{
15 Voir Darley Mathilde, «Le contrôle migratoire aux frontières Schengen : pratiques et représentations des polices
} sur la ligne tchéco-autrichienne », Cultures \& Conflits, n71, 2008, p. 13-29. 
« histoire plausible », c'est-à-dire un " récit imaginaire stéréotypé » ${ }^{16}$ (Heyman, 2009) composé à partir de critères plus ou moins subjectifs mêlant l'ethnicité supposée du voyageur, son âge, son genre, ou encore son « apparence $»^{17}$.

A ces «bricolages» quotidiens qui fabriquent la frontière sur sa scène d'exposition la plus évidente, le poste-frontière, je voudrais désormais ajouter l'étude de pratiques souvent pensées comme plus «discrètes » dans le tracé des frontières nationales, mais que les Etats les ont liées à la sécurisation de leurs frontières, à savoir les pratiques d'attribution de l'asile. Penser ensemble ces deux modalités de tracé des frontières de la communauté nationale permet non seulement d'insister sur la pluralité des formes et des métiers de la frontière, mais aussi de rendre compte d'une dynamique politique qui, à l'échelle européenne, a durci les conditions d'accès à l'asile en même temps que se renforçaient les contrôles aux frontières extérieures de l'Union ${ }^{18}$.

\section{"Mon rôle, c'est de juger si le migrant se comporte comme on attend de lui qu'il se comporte"}

Que nous apprennent les observations réalisées à la frontière aéroportuaire, et lors des entretiens conduits par les officiers de protection de l'asile (fonctionnaires des ministères de l'Intérieur) avec les demandeurs d'asile arrivés par avion et détenus plusieurs semaines dans un centre fermé jusqu'à ce qu'une décision soit rendue sur le bien-fondé de leur demande, dans le cadre d'une procédure dite « accélérée »?

Ces entretiens peuvent être vus comme le moment par excellence de confrontation du migrant à la frontière séparant le migrant «victime» de persécutions attestées et/ou perçues comme crédibles dans son pays d'origine et dont la présence apparaît «légitime», de la figure «illégitime » du « réfugié économique ». La mise à l'épreuve institutionnelle de son récit vise alors, pour les officiers de protection, à vérifier que celui-ci s'inscrit dans le cadre normatif prévu par l'institution, lequel consacre une forme standardisée de récit propre aux codes narratifs occidentaux $^{19}$, sur le fond comme sur la forme. Matérialisé par la trame écrite standardisée guidant les entretiens des officiers de protection avec les demandeurs d'asile, ce récit idéal-typique consacre à la fois le «règne du détail » et la capacité du requérant à articuler son histoire personnelle au «grand récit collectif $»^{20}$, associé par les offices nationaux de protection à chaque pays d'origine à partir des principaux faits marquants de son histoire sociale, religieuse et politique. Surtout, pour être cru, le récit doit entrer en résonance avec la catégorie du réfugié, pensée comme "pure »" évaluée à l'aune de la capacité du demandeur à donner à voir et à entendre les souffrances qu'il aurait subies et qui attesteraient de son statut de victime ${ }^{22}$. Les observations conduites lors des interrogatoires entre représentants de l'autorité étatique et

\footnotetext{
${ }^{16}$ Heyman Josiah McC., « Risque et confiance dans le contrôle des frontières américaines », Politix, vol. 3, n87, 2009, p. 21-46.

${ }^{17}$ Voir aussi Casella-Colombeau Sara, "Policing the internal Schengen borders: Managing the double bind between free movement and migration control", Policing and Society, vol. 27, n5, 2015, p. 480-493.

18 A l'échelle locale de la frontière considérée ici, on a vu se développer en Autriche et en République tchèque entre 2004 et 2007, dans un contexte de disparition programmée de la frontière Schengen qui les séparait, les possibilités de détention des demandeurs d'asile à des fins d'expulsion dès le rejet de leur demande.

${ }^{19}$ Bohmer Carol, Shuman Amy, Rejecting Refugees: Political Asylum in the 21st Century, London/New York, Routledge, 2007.

${ }^{20}$ D'Halluin Mabillot Estelle, Les épreuves de l'asile. Associations et réfugiés face aux politiques du soupçon, Paris, Éditions de l'EHESS, 2012.

21 Malkki Liisa H., Purity and Exile: Violence, Memory, and National Cosmology among Hutu Refugees in Tanzania, Chicago, The University of Chicago Press, 1995.

${ }^{22}$ Kobelinsky Carolina, L'accueil des demandeurs d'asile. Une ethnographie de l'attente, Paris, Le Cygne, 2010.
} 
demandeurs d'asile dans les centres fermés aéroportuaires révèlent que l'appréciation de la souffrance personnelle, dictant l'octroi d'une protection, est largement fonction des formes d'engagement compassionnel que le récit délivré parvient à susciter chez l'officier en charge de l'examen de la demande ${ }^{23}$. En l'absence d'instrument officiel de mesure de la souffrance et/ou de la «victimité », c'est alors souvent l'expérience personnelle de l'officier qui commande sa réception de «l'histoire » qui lui est présentée. Différentes situations d'entretiens témoignent ainsi du recours récurrent mais plus ou moins explicite, de la part des officiers, à la comparaison entre leur vécu familial, professionnel ou affectif et celui des demandeurs pour décider de la crédibilité à accorder à leur récit: "Mais enfin, ce n'est pas possible, moi j’ai toujours su combien gagnait mon père! [...] Nous, à la maison, nous avons toujours parlé de notre avenir avec mes parents...", déclare ainsi un officier de protection à un demandeur d'asile lors de l'examen de sa demande en centre fermé. Autrement dit, la légitimité octroyée à la demande s'adosse, en partie au moins, à l'accessibilité empathique du demandeur pour l'officier, elle-même évaluée à l'aune d'un «chez moi » ou «chez nous » fréquemment invoqué par les officiers pour rendre compte de conceptions supposées collectivement partagées «en Occident». Erigé en norme, ce "chez nous » dicte dès lors les «attentes» par un officier de protection à l'encontre des demandeurs; attentes dont on peut postuler qu'elles revêtent même ici, en commandant l'octroi ou non d'une protection, un caractère d' " exigences » à l'égard des requérants.

S’intéresser aux échanges entre demandeurs d'asile et officiers de protection ne permet donc pas tant ici d'analyser les décisions en matière d'asile, puisqu'aucune systématisation statistique n'a accompagné l'enquête ethnographique, que de saisir certains des éléments constitutifs d'un « récit d'asile légitime » pour les acteurs de la procédure et d'accéder ainsi aux représentations orientant leurs pratiques d'octroi ou de refus de la protection. Il apparait ainsi que, dans un contexte a priori juridiquement très normé, la décision d'octroi d'une protection n'est pas le seul fruit des normes procédurales guidant le travail des officiers, mais une production socialement, culturellement et même émotionnellement située.

\section{Faire avec, faire contre la frontière}

S'intéresser aux pratiques quotidiennes par lesquelles, en différents lieux, les agents étatiques en charge de garder les frontières distinguent les migrants « légitimes » des « illégitimes » invite donc à remettre en cause l'idée encore largement répandue de frontières nationales dont le tracé obéirait, de manière quasi mécanique, à l'application de principes strictement définis par l'Etat de droit et auxquels contreviendraient les candidats se voyant refuser l'entrée et/ou le séjour sur le territoire. Se dessinent, non pas une, mais des frontières prenant corps en une multitude de lieux et s'incarnant dans une diversité d'acteurs, et dont le tracé apparaît informé non seulement par l'appareil législatif des Etats, mais aussi par l'ensemble des normes extra-juridiques régissant le rapport des communautés nationales à l'« Etranger», normes dont les gardiens étatiques des frontières ne sont alors que les (ré)activateurs. Comprendre la frontière et ses effets dans leur fabrique quotidienne implique alors de prendre en compte l'ensemble des acteurs qui s'y côtoient, s'y opposent et/ou s'y évitent, et l'appareil de normes que chacun d'entre eux mobilise pour

\footnotetext{
${ }^{23}$ Darley Mathilde, «Le pouvoir de la norme. La production du jugement et son contournement dans les lieux d’enfermement des étrangers », Déviance et Société, vol. 34, n², 2010, p. 229-239.
} 
légitimer, mais aussi contester la frontière. A cet égard, il est particulièrement intéressant de se pencher sur les acteurs qui font précisément du droit un des outils de contestation et/ou de contournement des frontières étatiques, à savoir les acteurs non gouvernementaux en charge de l'assistance juridique aux étrangers placés en rétention. L'observation quotidienne de leurs pratiques d'accompagnement des étrangers montre en effet que, en marge de leur engagement dans la défense des droits des étrangers, leurs activités au sein de lieux-frontières les contraignent non seulement à reconnaître l'existence des frontières mais aussi, dans leurs tentatives de faire contre, à réaffirmer certaines des normes extra-juridiques qui en dessinent les contours. L'attention prêtée aux étrangers eux-mêmes et aux stratégies qu'ils déploient pour passer ou contourner les contrôles contribue par ailleurs aussi à remettre en cause ce mythe de la frontière comme point de clôture du territoire. Elle témoigne en outre de la possibilité d'une réappropriation, par les candidats au franchissement, des attentes normatives présidant à la légitimation de certains récits, réappropriation qui semble pouvoir également contribuer à la diffusion des stéréotypes fabriquant, au quotidien, la frontière.

\section{Assister les étrangers aux frontières nationales, une contestation du dispositiffrontalier?}

Ces acteurs non gouvernementaux intervenant dans les centres fermés pour étrangers en instance d'éloignement du territoire mettent généralement en avant une posture critique vis-à-vis des politiques d'enfermement et d'expulsion des étrangers. Ils sont souvent les seuls acteurs non policiers au contact des retenus dans les centres fermés, et de ce fait en position de recueillir une parole a priori détachée des injonctions institutionnelles. "Nous sommes de l'Eglise, pas de la police, vous pouvez nous parler sans crainte! » est ainsi la phrase par laquelle les aumôniers que j’ai accompagnés pendant plusieurs mois dans un centre de rétention allemand avaient coutume de se présenter aux étrangers rencontrés pour la première fois. Au-delà du rôle de médiateurs, voire d'agents pacificateurs entre les murs, ces acteurs non gouvernementaux, religieux ou non, ont également investi les voies de recours juridique contre la mesure d'enfermement et/ou d'expulsion, situant leur engagement humaniste et religieux au sein d'un dispositif répressif ${ }^{24}$. Pour ce faire, ils cherchent à repérer dans la biographie des étrangers retenus, au cours des entretiens qu'ils conduisent avec eux, les éléments pouvant être convertis en arguments juridiquement valables face à l'administration afin de contester la mesure d'éloignement du territoire. Autrement dit, ils font également office de « filtres » entre les étrangers enfermés et les dispositifs juridiques extérieurs qui permettent de contester l'éloignement du territoire. Bien qu'officiellement mandatés pour assurer l'accompagnement spirituel des étrangers retenus, les aumôniers protestants et catholiques suivis dans un centre de rétention allemand, dont la trajectoire professionnelle est souvent marquée par leur engagement en faveur des droits des étrangers, ont eux aussi investi les normes juridiques en l'absence d'autre acteur assurant une assistance juridique en rétention et résument ainsi l'ambiguité de leur position : "Nous avons un énorme pouvoir. C'est nous qui décidons si ces gens vont recevoir de l'aide ou pas. "Il s'agit donc de s'intéresser ici à la manière dont ces aumôniers, de même que les acteurs non gouvernementaux observés dans d'autres dispositifs d'enfermement, prennent part à la mise en œuvre différentielle de la politique d'expulsion en contribuant à tracer la frontière entre migrants « expulsables » et

\footnotetext{
${ }^{24}$ Darley Mathilde, «Le bon, la brute et le migrant ? Le rôle négocié des acteurs religieux dans un dispositif policier d'enfermement des étrangers en Allemagne », Sociologie du travail, n56, 2014, p. 472-492.
} 
migrants « intégrables $»^{25}$.

Les aumôniers ont la particularité, tout en mettant en œuvre une politique d'accompagnement juridique des retenus, de revendiquer précisément l'« empathie» comme compétence professionnelle dans un dispositif répressif, en l'opposant à la nature disciplinaire du travail policier. Or le suivi quotidien des intervenants religieux dans les cellules d'un centre de rétention allemand révèle que cette disposition à l'écoute ne s'accomplit pas de manière systématique avec l'ensemble des retenus. Alors que certains migrants deviennent, plusieurs semaines durant, des cibles privilégiées de la relation d'aide, d'autres au contraire semblent passer «à travers les mailles du filet ${ }^{26}$. Les observations révèlent ainsi que l'engagement des aumôniers dans la relation d'assistance dépend en grande partie de la capacité des étrangers placés en rétention à "performer" «un comportement émotionnellement en accord avec les stéréotypes du comportement "approprié" du réfugié $»^{27}$. Les attributs comportementaux des retenus guidant la propension des aumôniers à écouter et à juger crédible leur récit recoupent ainsi ceux qui contribuent généralement à définir la figure stéréotypée de la «victime », à savoir l'expression de la gratitude, une attitude dépressive, le fait de présenter peu d'exigences dans la relation aux autorités, mais aussi certaines formes de "compliance» avec le travail associatif, notamment la disposition à s'engager avec «ferveur » dans les activités de loisirs et/ou religieuses proposées en rétention ; autant de caractéristiques semblant faire du retenu un interlocuteur émotionnellement plus accessible, et partant juridiquement plus «défendable». A cet égard, les femmes détenues, particulièrement ciblées par les activités de loisirs organisées en rétention par les aumôniers, semblant y voir de « saines » occupations « féminines » (découpages, préparations de décorations de Noël et ateliers culinaires notamment), sont explicitement présentées par les acteurs religieux, hommes et femmes, comme plus susceptibles de répondre aux attentes comportementales en raison de leur caractère décrit comme plus sensible, plus fragile et plus soumis, et du «besoin particulier de protection » qui en découlerait. Autrement dit, conformément à la représentation dominante dans les politiques d'immigration, mais aussi de lutte contre les violences faites aux femmes, la femme migrante serait intrinsèquement " plus victime » que l'homme. Corrélé à cette représentation, un lien est directement établi entre parcours migratoire féminin et victimité. Émergent alors, dans les pratiques quotidiennes d'assistance religieuse en rétention, deux figures idéal-typiques de la «vulnérabilité » : la femme victime de violences conjugales, d'une part, et la femme victime de la traite à des fins d'exploitation sexuelle d'autre part. Cependant, si ces figures apparaissent en tout point conformes à la représentation dominante de l'ordre des sexes faisant de la violence un attribut du masculin viril, l'accession des femmes étrangères placées en rétention à la qualification de «victimes » apparaît plus ou moins systématique en fonction de leur origine. Les logiques de genre croisent en effet ici des logiques d'appartenance supposée à une nation et/ou à un groupe ethnico-racial. Les migrantes auxquelles on prête une appartenance «rom » sont ainsi généralement décrites comme victimes de violences conjugales, tandis que les migrantes africaines sont quasi-systématiquement associées par les aumônières à la traite à des fins d'exploitation sexuelle: «pour l'Afrique, je pars du principe qu'elles sont presque toutes

\footnotetext{
${ }^{25}$ Fischer Nicolas, «Une frontière "négociée". L'assistance juridique associative aux étrangers placés en rétention administrative », Politix, vol. 22, n³, 2009, p. 71-92.

${ }^{26}$ Darley Mathilde, «Les coulisses de la nation » Assignations genrées et racialisées dans les pratiques d'assistance aux étrangers en situation irrégulière, Sociétés contemporaines, 2014, vol. 2, n 94, p. 19-40 .

${ }^{27}$ Graham Mark, «Emotional Bureaucracies : Emotions, Civil Servants, and Immigrants in the Swedish Welfare State », Ethos, vol. 30, n³, 2002, p. 199-226.
} 
victimes de la traite », m'explique ainsi l'une d'entre elles. Les détenues vietnamiennes sont au contraire généralement décrites comme "renfermées », trait de caractère qui justifierait leur moindre inclusion dans le maillage assistanciel en rétention. Autrement dit, les pratiques d'intervention des acteurs non gouvernementaux en rétention s'adossent également à des raisonnements par groupes et par stéréotypes reposant pour partie au moins sur une anticipation des chances de succès des recours juridiques, mais reproduisant de ce fait largement les « histoires plausibles » construites par les institutions de contrôle : celles à propos desquelles un consensus compassionnel (dont les ressorts genrés et racialisés méritent d'être interrogés) parait pouvoir émerger avec les professionnels de la frontière, institutionnels et policiers.

\section{Passer les frontières : migrants et passeurs aux marges de l'Europe}

Face à la prégnance de la pensée institutionnelle par groupes ainsi que des ressorts émotionnels activés par le stéréotype de la "victime» et des représentations racialisées et genrées qui le nourrissent, dans quelle mesure les étrangers demandant la reconnaissance de leur légitimité à séjourner sur le territoire peuvent/doivent-ils s'y conformer ou, au contraire, chercher à les contourner? Les représentations dominantes, étatiques et non étatiques, concourant à faire de la victime méritante et reconnaissante la seule figure audible semblent d'abord conduire à un investissement particulier des registres de la supplique et de la charité de la part des étrangers cherchant à inscrire leur récit de vie dans le cadre normatif véhiculé par l'institution ${ }^{28}$. Cette tendance se trouve accentuée par les politiques migratoires contemporaines qui, après avoir considérablement restreint les possibilités d'immigration légale depuis les années 1970, ont contribué à faire de la demande d'asile l'une des dernières voies légales de franchissement des frontières et/ou de contournement de l'expulsion. Au cours des années 1990, on observe alors dans la plupart des pays européens une suspicion croissante à l'égard des demandeurs d'asile soupçonnés d'«abuser» de la procédure pour conférer une dimension humanitaire et/ou politique à des migrations dites « économiques »; rhétorique faisant fi de la complexité même des mouvements de personnes mêlant le plus souvent des motivations de différentes natures. Ce discours justifie néanmoins, depuis les années 2000 notamment, l'augmentation des possibilités légales de placer les demandeurs d'asile en rétention, alors même que la demande d'asile est ellemême devenue l'un des principaux moyens d'éviter ou de retarder, en rétention, une expulsion devant intervenir rapidement dans un contexte de relative rareté des ressources juridiques permettant sa contestation. Autrement dit, les lieux-frontières apparaissent à la fois comme des espaces où se trouvent matérialisées les restrictions aux canaux d’immigration légale, et comme des espaces de production du «demandeur d'asile» en favorisant l'activation par les migrants d'une "rhétorique de l'asile ». Animée par la recherche du conformisme aux normes véhiculées par l'institution (ou ce qu'ils en perçoivent), cette rhétorique se distingue notamment par la mise en avant de la condition de «victime » du demandeur, comme l'illustre le témoignage implorant d'Ousmane, enregistré à sa demande, seul dans sa chambre.

"Je suis un Malien, un homme du Mali. J'ai quand même pas eu le temps d'expliquer à tout un chacun mais cette fois-ci je veux éclaircir quelques points de mon passé. J'ai été victime dans mon enfance dans mon pays natal au Mali de la perte de mes parents. Mes parents, c'est-à-dire, ont été assassinés quand j'étais tout petit...

\footnotetext{
${ }^{28}$ Fassin Didier, «Charité bien ordonnée. Principes de justice et pratiques de jugement dans l'attribution des aides
} d'urgence », Revue française de sociologie, vol. 42, n³, 2001, p. 437-475. 
Certainement, par après, à travers mon évolution, quelque part dans Bamako, j'ai eu la chance de connaître un certain Anglais, un certain Monsieur Richard. [...] J'ai quitté le pays à travers ce monsieur, comme destination Angleterre, pour le but d'avoir de l'emploi, de pouvoir survivre. On est venus jusqu'en République tchèque, il a disparu avec tous mes dossiers, toutes mes papiers, avec mon argent, il m'a trabi [...] Impossible de se retourner moi dans mon pays natal, impossible, tout à fait impossible. [...] J'aurais même préféré d'être enterré vivant dans mon coin ici au lieu d'être renvoyé, au lieu de rentrer dans le pays natal. Ça veut dire que c'est impossible. La République tchèque vraiment est tout à fait... géniale, tout à fait généreux. C'est tout. Etant donné les organisations qui sont là, qui aident les réfugiés, nous en suis reconnaissants envers ceux-là, et que nous les félicitons, nous leur encourageons de plus. »

Face à la politique du soupçon entourant les demandes d'inclusion sur le territoire national, ce témoignage cherche à susciter l'émotion et la compassion de l'auditeur éventuel en inscrivant le récit dans le registre de la «supplique » et de la présentation de soi comme «bonne victime ». Ces tentatives émergent d'autant plus que, dans les premiers flottements de mon arrivée dans un centre policier fermé en tant que bénévole pour une association, je suis alors encore assimilée, par Ousmane, à un acteur susceptible d'intercéder en sa faveur auprès des autorités compétentes. Le récit enregistré ici part ainsi des épreuves justifiant le départ, pour évoquer ensuite la tromperie de la part du passeur, et glisser enfin vers la reconnaissance et à la gratitude témoignée à l'institution et au pays d'accueil. Il est par ailleurs intéressant de constater que, dans un contexte où les politiques de lutte contre l'immigration irrégulière justifient désormais régulièrement la nécessité du « retour des frontières » par celle de mettre fin à l'activité de réseaux de passeurs sans scrupules exploitant la « misère humaine » (ce qui revient à conférer une légitimité « humanitaire » à la dimension répressive du contrôle), les migrants eux-mêmes invoquent fréquemment cette figure de «victimes des passeurs » dans les récits qu'ils délivrent lors de nos premières rencontres. L'argumentaire humanitaro-répressif dénonçant dans un même mouvement l'immigration irrégulière et l'exploitation des migrants par les passeurs semble ainsi pénétrer les techniques de présentation de soi des demandeurs d'asile et la mise en récit à la fois de leur condition de victime et de leur légitimité à prétendre au statut de réfugié. Cependant, alors que ma présence dans les lieux d'enfermement se prolonge et que mon statut extérieur à l'institution étatique se confirme, les récits se font plus différenciés et les recours à la rhétorique "victimisante » de l'institution moins fréquents. Les "problèmes» d'abord invoqués s'estompent et le souhait pieux de l'intégration en République tchèque est remplacé par l'exposition des possibilités de passage vers les pays membres de l'UE situés plus à l'Ouest. En conséquence, la place du passeur dans les discours recueillis évolue également : il n'est plus tant «l'exploiteur» dont le manque de scrupule justifie, en partie au moins, le statut de victime du migrant et son besoin de protection, qu'un acteur indispensable du voyage dont la légitimité et les tarifs ne sont plus contestés. On n'est plus en présence de deux «figures antithétiques [...] séparées pour les besoins de la morale », le migrant victime et le passeur abject, mais il s'agit au contraire de la «figure complexe d'un phénomène unique : celui de tout départ forcé [...] effectué en toute méconnaissance du bon itinéraire illégal pour arriver sain et sauf dans un pays aussi sûr que possible ${ }^{29}$. Autrement dit, certaines formes de mise en récit «standardisées» de leur trajectoire migratoire par les demandeurs d'asile reposant sur la victimisation et l'humanitarisation de leur cause peuvent être

\footnotetext{
${ }^{29}$ Voir Laacher Smaïn, Mokrani Laurette, «Passeur et passager : deux figures inséparables », Plein Droit, 55, 2002, disponible sur http://www.gisti.org/doc/plein-droit/55/passeur.html (23/10/2007).
} 
vues à la fois comme le résultat d'un appel à la mémoire artificiellement provoqué $e^{30}$ par l'institution, et comme le fruit d'une intériorisation, par les candidats à la protection, des normes extra-juridiques émaillant la manière dont est « dit» le droit d'asile.

Les passeurs eux-mêmes, ou en tout cas ceux avec lesquels il m'a été possible de m'entretenir, recourent également à la justification humanitaire de leur intervention dans l'organisation du voyage clandestin. Johnny, ancien policier congédié pour trafic de drogues et reconverti dans le convoi de migrants en situation irrégulière depuis la République tchèque vers l'Italie, énonce ainsi :

"Le but premier est bien sûr toujours de gagner de l'argent. Mais le groupe avec lequel je travaillais avait aussi pour priorité d'assurer la sécurité non seulement des membres du groupe eux-mêmes mais aussi des gens qu'ils faisaient passer, tout en n'étant pas chers. Nous les emmenions vers la liberté. Sous le communisme aussi, je faisais passer des gens, et à l'époque c'était considéré comme hérö̈que par une majorité de la population. Aujourd'bui c'est pareil, j'essaye aussi de faire passer des gens pour qu'ils puissent enfin être libres ».

Les étrangers détenus aux frontières insistent ainsi souvent sur le fait de n'avoir commis aucun autre crime que celui de "vouloir être libre», soif de liberté qui conduit précisément à leur placement en rétention. L'accent mis sur la contradiction existant entre leur enfermement par les démocraties occidentales et les valeurs prônées par ces dernières constitue également un moyen de miner la légitimité de la frontière et des lieux de sa matérialisation.

"C'est pire que Guantanamo ici, vous voyez, ces grilles devant les fenêtres? Deux épaisseurs de grilles devant les fenêtres! Comme si on était des criminels! On devrait avoir vraiment commis un crime, au moins on saurait pourquoi on est ici. L'Autriche se dit un pays démocratique mais ce n'est pas une démocratie ici, on ne respecte pas les droits de l'homme, le sang d'Hitler coule encore dans leurs veines, ils n'aiment pas les Noirs. [...] Mandela s'est battu pour la fin de l'apartheid en Afrique, mais ce qui se passe ici ce n'est pas autre chose: c'est du racisme moderne ».

De même, en se référant fréquemment aux violations des droits de l'homme généralement associées à leur pays d'origine et dont ils ont pu être victimes, et en inscrivant dans le prolongement de ces souffrances passées la répression à laquelle les soumettent les démocraties de l'Ouest, les migrants établissent un parallèle entre ces dernières et les régimes politiques, africains notamment, sur lesquels les démocraties n’ont eu de cesse de rappeler leur supériorité morale. Ce retournement du stigmate fait alors du migrant le dépositaire de valeurs morales universalistes, parmi lesquelles la liberté humaine, tandis que l'Etat, qui ferme ses frontières tout en tirant sa légitimité de la proclamation du respect de principes démocratiques, est érigé en « transgresseur » d'un ordre moral et politique universel.

Enfin, la remise en cause du pouvoir institutionnel d'identification et de «tri » des populations dans les lieux d'enfermement des étrangers passe également par le déni d'information opposé par certains migrants aux institutions étatiques de contrôle des frontières, les privant de fait de leur pouvoir d' «étiquetage » et de tri des circulations. En effet, tant que la traçabilité biométrique du migrant n'est pas assurée (c'est-à-dire tant que ses empreintes et les informations le concernant n'ont pas encore été enregistrées et partagées par l'une des polices européennes), l'éloignement n'est par définition pas possible. Dès lors, ne rien dire, donner une fausse identité, ou déclarer être originaire d'un pays connu pour les problèmes de coopération qu'il pose dans le processus de

\footnotetext{
${ }^{30}$ Pestre Elise, La vie psychique des réfugiés, Paris, Payot et Rivages, 2010.
} 
reconnaissance et de réadmission de ses ressortissants, sont autant de «contre-conduites » permettant de mettre à distance l'emprise institutionnelle et d'éviter notamment l'expulsion. De même, se prétendre ressortissant d'un Etat dont le système d'enregistrement des naissances à l'Etat civil est réputé défaillant et dont l'histoire collective a été associée, dans le "grand récit» reconstitué par les institutions d'attribution de l'asile, à la production de « victimes » (tel le Sierra Leone et ses « enfants-soldats » à l'époque de notre enquête), peut constituer une ressource face à la pensée par groupes mise en place par les offices de protection.

"Nous expulsons de moins en moins de personnes chaque année tout simplement en raison de problèmes juridiques et pratiques. Parce que nous ne savons pas qui nous avons en face de nous. Et je n'exagère pas! S'il dit qu'il s'appelle Mickey Mouse, [...] tant que nous ne pouvons pas apporter la preuve qu'il ne s'appelle pas Mickey Mouse, il a gagné. »

Ce dernier extrait d'un entretien avec un responsable policier, et les observations consignées plus haut, n'ont évidemment pour objet ni de questionner les besoins de protection des demandeurs d'asile, ni de légitimer les affirmations des représentants des institutions de contrôle selon lesquelles les lieux d'enfermement (centres fermés pour demandeurs d'asile et centres de rétention) seraient peuplés quasi exclusivement de «faux réfugiés ». Tout au plus permettent-elles tenter de complexifier l'histoire dominante de l'Etat fort imposant ses frontières en rendant compte non seulement des pratiques de contrôle, mais aussi des stratégies d'ajustement et/ou d'évitement qu'elles génèrent chez les migrants, leur conférant des capacités d'action, fussentelles limitées, dans la mise en œuvre des frontières.

Les enquêtes auprès de ceux qui font et défont quotidiennement la frontière dans les lieux de contrôle de l'immigration invite donc à sortir de l'appréhension de ces lieux-frontières au seul prisme du contrôle, pour envisager la multiplicité des acteurs présents et leurs rôles respectifs dans la pratique des frontières. Plutôt que d'opposer agents du contrôle et agents de sa contestation dans un face-à-face irréconciliable, l'observation de la fabrique locale des frontières nationales dessine différentes pratiques, étatiques et non étatiques, constitutives de la "patrouille des frontières $»^{31}$. Autrement dit, plus qu'un espace où l'autorité souveraine d'exclure s'appliquerait mécaniquement, la frontière apparait comme le lieu de bricolages quotidiens mêlant la norme juridique à d'autres ensembles de normes et de représentations traduisant avant tout l'incapacité des acteurs à s'extraire d'une pensée de l'étranger par sa supposée altérité. Le consensus compassionnel émergeant autour de la figure de la «bonne victime», souffrante, méritante et reconnaissante, entre des acteurs affichant pourtant des finalités radicalement différentes quant au gouvernement des frontières, témoigne ainsi de la prégnance d'une appréhension de l'Autre dans un cadre interactionnel profondément inégalitaire : pour apparaittre légitime, le migrant doit d'abord fournir la preuve de son caractère ostensiblement plus malheureux et plus fragile que les représentants de la communauté nationale auxquels il expose son récit ${ }^{32}$. L'altérisation fondamentale à laquelle conduit la politique compassionnelle et la

\footnotetext{
31 Bilge Sirma, «"... alors que nous, Québécois, nos femmes sont égales à nous et nous les aimons ainsi" : La patrouille des frontières au nom de l'égalité de genre dans une «nation » en quête de souveraineté », Sociologie et sociétés, 421, 2010, p. 197- 226.

${ }^{32}$ Fassin Didier, La Raison humanitaire. Une histoire morale du temps présent, Paris, Éditions de l'EHESS, 2010.
} 
pensée par groupes se double cependant d'une exigence de mimétisme adressée à l'étranger dans la délivrance de son récit censé reproduire les cadres narratifs institutionnels tout en témoignant de son accessibilité émotionnelle. C'est ce paradoxe, entre injonction à la différence et exigence de reproduction des cadres cognitifs et comportementaux connus et maîtrisés, que vient nourrir la légitimation «humanitaro-répressive » du contrôle qui gagne du terrain depuis une dizaine d'années en Europe. Plus qu'une zone de réaffirmation par l'Etat de ses prérogatives régaliennes, la frontière apparaît alors comme une zone d'inconfort, mettant en lumière, depuis les marges, les contradictions des politiques migratoires contemporaines. 